\title{
Enhanced cAMP-stimulated protein kinase $A$ activity in human fibrolamellar hepatocellular carcinoma
}

\author{
Kevin M. Riggle ${ }^{1}$, Kimberly J. Riehle ${ }^{1,2,3,4}$, Heidi L. Kenerson', Rigney Turnham ${ }^{5,6}$, Miwako K. Homma ${ }^{5,7}$, Machiko Kazami', \\ Bret Samelson ${ }^{5,6}$, Renay Bauer ${ }^{1}$, G. Stanley McKnight ${ }^{6}$, John D. Scott ${ }^{5,6}$ and Raymond S. Yeung ${ }^{1,2}$
}

BACKGROUND: Fibrolamellar hepatocellular carcinoma (FL-HCC) affects children without underlying liver disease. A consistent mutation in FL-HCCs leads to fusion of the genes encoding a heat shock protein (DNAJB1) and the catalytic subunit of protein kinase $A$ (PRKACA). We sought to characterize the resultant chimeric protein and its effects in FL-HCC.

METHODS: The expression pattern and subcellular localization of protein kinase A (PKA) subunits in FL-HCCs were compared to paired normal livers by quantitative polymerase chain reaction $(\mathrm{QPCR})$, immunoblotting, and immunofluorescence. PKA activity was measured by radioactive kinase assay, and we determined whether the FL-HCC mutation is present in other primary liver tumors.

RESULTS: The fusion transcript and chimeric protein were detected exclusively in FL-HCCs. DNAJB1-PRKACA was expressed 10-fold higher than the wild-type PRKACA transcript, resulting in overexpression of the mutant protein in tumors. Consequently, FL-HCCs possess elevated CAMP-stimulated PKA activity compared to normal livers, despite similar $\mathrm{K}_{\mathrm{m}} \mathrm{s}$ between the mutant and wild-type kinases.

CONCLUSION: FL-HCCs in children and young adults uniquely overexpress DNAJB1-PRKACA, which results in elevated CAMPdependent PKA activity. These data suggest that aberrant PKA signaling contributes to liver tumorigenesis.

$\mathrm{H}$ epatocellular carcinoma (HCC) is one of the most common cancers worldwide, and is the third leading cause of cancer death (1). Multiple HCC risk factors have been identified, including chronic infection with hepatitis B or $\mathrm{C}$ virus, alcoholic cirrhosis, nonalcoholic steatohepatitis, and exposure to aflatoxin (2). HCC is a genetically heterogeneous disease, as evidenced by these varied risk factors and the resultant underlying cirrhosis that predisposes the liver to carcinogenesis (2-4). Fibrolamellar HCC (FL-HCC) is a subtype of HCC that is not only clinically and histologically homogenous, however, but also occurs in children and young adults without underlying liver disease $(5,6)$. Histologically, FL-HCCs contain pale, pleomorphic hepatocytes with abundant eosinophilic cytoplasm arranged within a network of lamellar collagen fibers, for which FL-HCC was named.

With recent improvements in whole genome and exome sequencing, there have been significant breakthroughs in our understanding of the molecular pathogenesis of FL-HCC. Honeyman et al. (7) sequenced a series of FL-HCCs and discovered that all tumors in their cohort have a deletion in chromosome 19, which results in a chimeric transcript that contains the 5 ' region (exon 1) of the gene-encoding heat shock protein 40 (DNAJB1) fused in-frame to exon 2 of the catalytic (C) subunit of protein kinase A (PRKACA). Tumors express both the chimeric protein (DNAJ-PKA C) and the wild-type (WT) kinase, and the mutation is not found in adjacent normal liver from the same patients, suggesting that it is somatic in nature. Recently, Xu et al. (8) demonstrated that over expression of the chimeric gene enhances proliferation and colony formation in a transformed hepatocyte cell line, supporting its function as an oncogene. Moreover, structural studies have shown that the DNAJ-PKA C fusion protein folds correctly to form a mutant enzyme with all of the hallmarks of an active protein kinase (9). Several key questions remain, however, including: (i) is this mutation unique to FL-HCC or is it found in other primary liver tumors, (ii) is PKA activity enhanced in FL-HCC compared to nontumor livers, and (iii) does the mutant PKA C subunit have similar enzymatic properties as the WT C subunit? PKA normally exists in the cytoplasm as a holoenzyme composed of two PKA C subunits and two regulatory (R) subunits, of which there are four isoforms $(\mathrm{RI} \alpha, \mathrm{RI} \beta$, RII $\alpha$, RII $\beta)(10,11)$. The $\mathrm{R}$ subunits function to keep the $\mathrm{C}$ subunits inactive in the absence of cyclic adenosine monophosphate (cAMP), and associate with A-kinase anchoring proteins (AKAPs) to constrain localization and function of the enzyme $(12,13)$. In the presence of cAMP, C subunits are released from $\mathrm{R}$ subunits and are thus able to phosphorylate PKA substrates to direct downstream signaling.

We sought to further characterize the DNAJ-PKA C chimeric protein and components of the PKA holoenzyme in FL-HCCs and paired normal liver samples from our institution. We

\footnotetext{
'Department of Surgery, University of Washington, Seattle, Washington; ${ }^{2}$ Northwest Liver Research Program, University of Washington, Seattle, Washington; ${ }^{3}$ Department of Pathology, University of Washington, Seattle, Washington; ${ }^{4}$ Division of General and Thoracic Surgery, Seattle Children's Hospital, Seattle, Washington; ${ }^{5} \mathrm{Howard}$ Hughes Medical Institute, University of Washington, Seattle, Washington; ${ }^{6}$ Department of Pharmacology, University of Washington, Seattle, Washington; ${ }^{7}$ Department of Biomolecular Science, Fukushima University School of Medicine, Fukushima, Japan Correspondence: Raymond S. Yeung (ryeung@uw.edu)

Received 19 March 2015; accepted 18 December 2015; advance online publication 30 March 2016. doi:10.1038/pr.2016.36
} 
confirmed that expression of the mutant transcript and protein is unique to FL-HCCs, and that tumors have enhanced PKA activation in response to cAMP. These findings have implications for the development of therapeutic interventions for patients with FL-HCC.

\section{RESULTS}

\section{Unique Expression of Mutant mRNA and Protein in Human FL-HCC}

We initially sought to determine whether the expression of DNAJB1-PRKACA is unique to FL-HCC. Quantitative realtime polymerase chain reaction (qRT-PCR) was performed on cDNA prepared from four FL-HCCs and paired liver samples, as well as nine "classic" HCCs and two cholangiocarcinomas (CC). Using primers spanning exon 1 of DNAJB1 and exon 3 of PRKACA that specifically detect the mutant transcript, we detected its expression only in FL-HCC samples; not in paired nontumor livers or other primary liver cancers (Figure 1, panel a). In one case where both a FL-HCC and a focal nodular hyperplasia were resected from the same patient, genomic sequencing using the UW-Oncoplex multiplex gene sequencing panel (14) revealed the chromosome 19 mutation in the FL-HCC, but not in the focal nodular hyperplasia or the patient's nontumor liver. This latter finding was of particular interest, since focal nodular hyperplasias can resemble FL-HCCs on cross-sectional imaging, in that both are characterized as hypervascular lesions with a central scar.

Next, we compared the relative mRNA expression of WT and mutant PRKACA in four FL-HCC tumor samples and paired nontumor livers. PCR was performed using primers that span exons 5-6 of PRKACA. We found that FL-HCC samples express some form of PRKACA at levels 10.6 \pm 2.1 -fold higher than paired nontumor livers $(P=0.016$, Figure 1 , panel $\mathbf{b})$. We also used allele-specific primers to compare the relative expression of WT PRKACA (exons 1-3) and WT DNAJB1 (exons 2-3) with that of DNAJB1-PRKACA (using primers as in Figure 1a) in FL-HCCs and paired normal livers. Expression levels of WT PRKACA and WT DNAJB1 in tumor samples were equivalent to those of paired, normal livers (Figure 1, panel c), despite the fact that FL-HCCs only have one WT allele of each of these genes. Further, WT DNAJB1 expression was three-fold higher than WT PRKACA expression, both in FL-HCCs and in paired normal livers. Interestingly, tumor-specific DNAJB1-PRKACA expression was 10.9 \pm 1.6 -fold higher than WT PRKACA and $3.8 \pm 1.1$-fold higher than WT DNAJB1. Together, these findings confirm that the mutant transcript is unique to FL-HCC, and that it is expressed at a significantly higher level than either WT PRKACA or WT DNAJB1.

Next, we used immunoblot analyses to determine relative expression of the DNAJB1-PRKACA fusion protein product (DNAJ-PKA C) and WT PKA C in FL-HCC and other primary liver tumors using an antibody directed against the C-terminus of PKA C. Two bands at approximately 46 and $41 \mathrm{kDa}$ are detected in all FL-HCC samples, corresponding to the fusion protein and WT PKA C, respectively (Figure 1, panel d). In contrast, only the $41-\mathrm{kDa} \mathrm{WT}$ form is detected in the paired, non-tumor livers (N) and in 10 "classic" HCC and 2 CC samples (Figure 1, panel e). Using densitometry analysis and normalizing to $\beta$-actin, we calculated that total PKA C (mutant and WT) protein levels are on average 2.6 \pm 0.5 -fold higher in FL-HCC samples compared to the WT protein found in paired nontumor livers (Figure 1, panel f). These findings support our qRT-PCR analyses demonstrating higher PKA C expression in FL-HCCs.

\section{Regulatory Subunit RNA and Protein Expression in Human FL-HCC}

PKA normally exists as a tetrameric holoenzyme composed of two C and two R subunits $(10,11)$; therefore, it is important to establish whether the DNAJ-PKA C fusion enzyme produced in FL-HCC can interact with the R subunits. We also determined whether $\mathrm{R}$ subunit expression levels are differentially regulated in FL-HCC. We found that at the mRNA

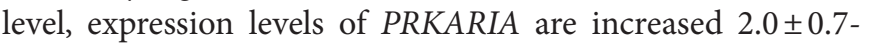
fold in FL-HCC samples compared to paired nontumor livers (Figure 2, panel a). At the protein level, the expression of PKA $\mathrm{RI} \alpha$ is $2.4 \pm 0.5$-fold greater in FL-HCC samples compared to nontumor liver (Figure 2, panels b,c). In contrast, PKA RII $\alpha$ mRNA and protein levels do not significantly differ between FL-HCC and nontumor liver samples, while PKA RII $\beta$ mRNA (Figure 2, panel a) and protein (Figure 2, panels b,c) are expressed at levels 1.7- and 2.4-fold lower in FL-HCCs compared to nontumor livers, respectively (Figure 2, panels b,c).

To determine whether DNAJ-PKA C can interact with physiological binding partners in a manner reminiscent of the WT protein, we performed immunoprecipitation in tumor and paired nontumor liver lysates using antibodies to the PKA RI $\alpha$ and PKA RII $\alpha$ subunits, followed by immunoblotting with antibodies against the $\mathrm{C}$-terminus of PKA C. Immunoblots with antibodies to PKA RI $\alpha$ and PKA RII $\alpha$ are shown as controls. Figure 3, panel a shows that both WT and mutant PKA $\mathrm{C}$ are present in the immunoprecipitates generated using both PKA R subunit antibodies, indicating that endogenous mutant PKA C can interact with both $\mathrm{R}$ subunits. This implies that RI and RII holoenzymes containing the mutant PKA C can form in the tumor cells. Based on relative band intensities, there is a possibility that PKA RI $\alpha$ preferentially binds the mutant PKA C subunit, while PKA RII $\alpha$ interacts equally with mutant and WT PKA C (Figure 3, panel a). However, it is difficult to make definitive conclusions about differential binding, as baseline expression levels of the PKA R subunits differ, and factors including cAMP levels and the availability of protein kinase A inhibitor (PKI) and AKAPs may contribute to these binding interactions. Further, after immunoprecipitating with RI $\alpha$ antibody, we did not detect RII $\alpha$ by immunoblot; nor did we detect RI $\alpha$ in our immunoprecipitates using RII $\alpha$ antibody (Figure 3, panel a). These findings are consistent with a wealth of cell based and structural data showing that each $\mathrm{R}$ subunit forms homo dimers through a four helix bundle motif at the extreme amino terminus of each $\mathrm{R}$ subunit protomer, precluding the formation of $\mathrm{RI} \alpha / \mathrm{RII} \alpha$ heterodimers within a single PKA holoenzyme complex (15-20). We performed an 


\section{Articles $\mid$ Riggle et al.}
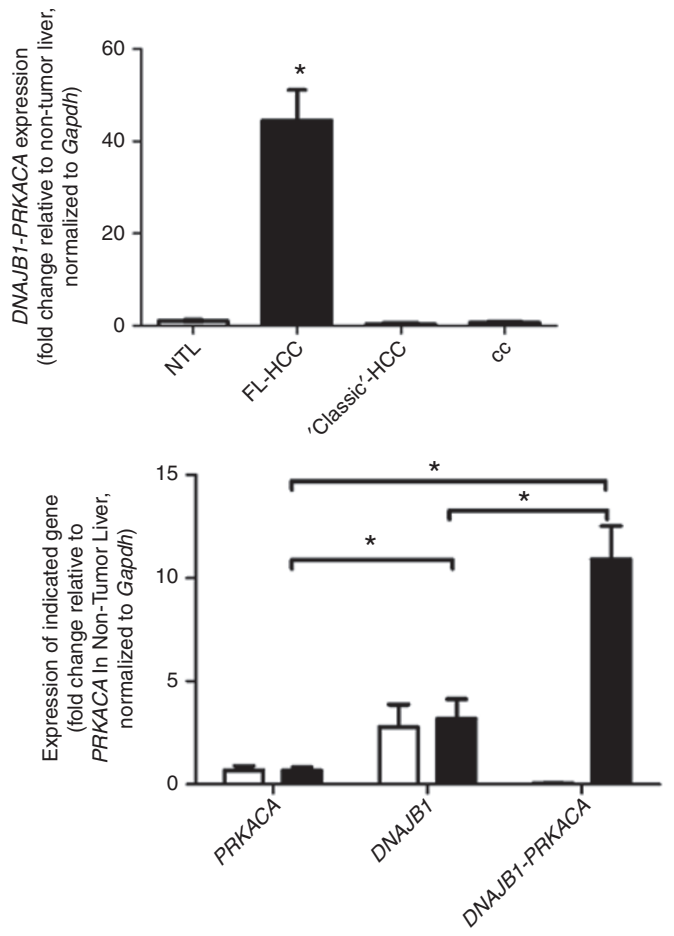

b

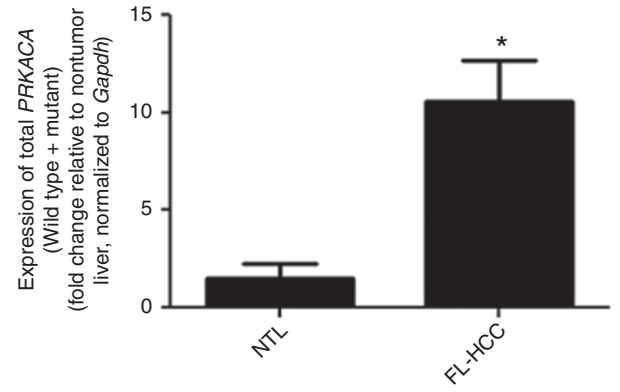

d

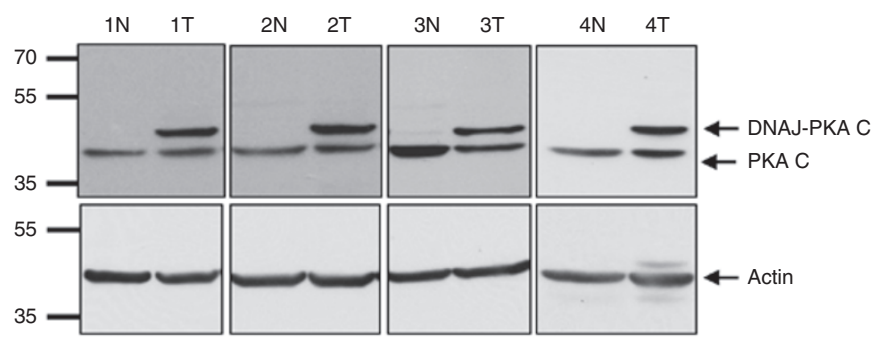

e
$\mathrm{N} \mathrm{HCC} \mathrm{CC} \mathrm{CC}$ $-\mathrm{HCC}-$
FL-HCC

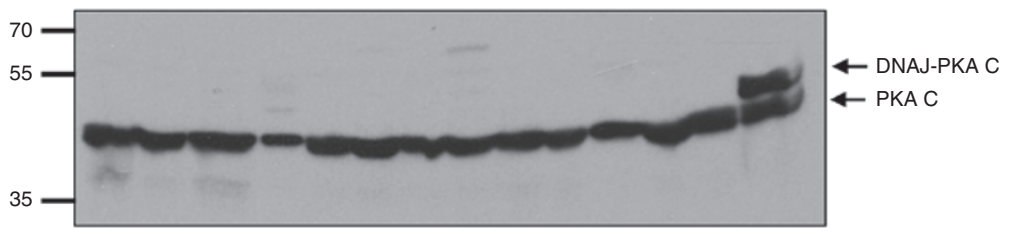

f

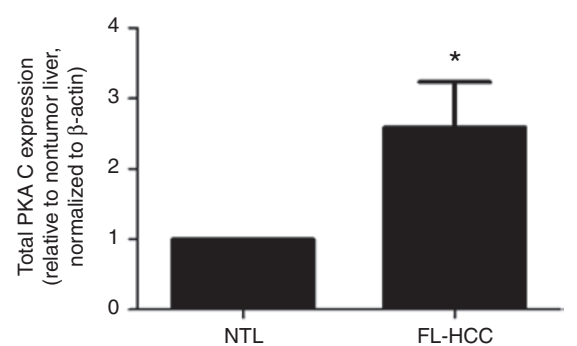

Figure 1. Unique RNA and protein expression of DNAJB1-PRKACA in FL-HCC. (a) Relative transcript expression levels of DNAJB1-PRKACA in FL-HCCs ( $n=$ 4), classic HCCs $(n=9)$, cholangiocarcinomas $(C C, n=2)$ compared to nontumor liver (NTL, $n=4)$. Results of qRT-PCR are normalized to Gapdh. ${ }^{*} P<0.05$ compared to all other groups. (b) Relative mRNA expression of WT and mutant $P R K A C A$ (total) in FL-HCCs compared with paired NTL. ${ }^{*} P<0.05$ comparing FL-HCC to NTL. (c) Relative expression of WT PRKACA, DNAJB1, and DNAJB1-PRKACA transcripts in FL-HCCs ( $n=4$, black bars) and paired, nontumor livers $(n=4$, white bars). Expression levels of PRKACA, DNAJB1 and DNAJB1-PRKACA were normalized to Gapdh, and are shown relative to WT PRKACA levels. ${ }^{*} P<0.05$ comparing DNAJB1-PRKACA to all other groups. (d) Immunoblot analyses of paired FL-HCC (T) and nontumor liver (N) samples from four patients showing expression of WT PKA C and mutant DNAJ-PKA C using an antibody against the $C$ terminus of PKA C. Actin expression is shown as loading control. (e) Immunoblot of classic HCCs and cholangiocarcinomas (CC) for PKA C. Normal liver (N) and FL-HCC are shown as controls. (f) Densitometry analysis of the immunoblot shown in (d) using Image J software (NIH), highlighting total PKA C (mutant plus wild type) protein expression in FL-HCC $(n=4$, black bar) vs. paired, nontumor liver $\left(n=4\right.$, white bar) ${ }^{*} P<0.05$. HCC, hepatocellular carcinoma; PKA, protein kinase $A$.

additional set of experiments using HEK293 cells that were transiently transfected by YFP-tagged WT and/or mutant PKA C, and confirmed that intact holoenzymes formed with mutant PKA C subunit were able to associate with the anchoring proteins Gravin and AKAP79 (Figure 3, panel b). Taken together, these studies suggest that the mutant DNAJ-PKA C fusion enzyme behaves like its WT kinase counterpart in terms of its ability to associate with R subunits and AKAPs.

\section{PKA Activity in Human FL-HCC}

The ability of the mutant PKA C to interact with PKA R subunits suggests that its kinase activity in FL-HCCs retains its dependency on cAMP, since cyclic nucleotide binding to the $\mathrm{R}$ subunits is a key element of PKA holoenzyme activation. To determine PKA activity in these cancers, we performed kinase activity assays using Kemptide as a substrate. These activity measurements were conducted on lysates from FL-HCCs and paired nontumor liver samples (21). Basal PKA activity (per microgram of protein) does not significantly differ in three of four FL-HCC samples compared to paired normal livers (Figure 4, panel a). Yet, in one sample (\#4) there is a slight but significant increase in basal PKA activity in the FL-HCC compared to the paired, nontumor liver ( 0.11 vs. $0.02 \mathrm{pmol} /$ $\mathrm{min} / \mathrm{mg}, P=0.01$. We then measured PKA activity in the presence of cAMP, which leads to release of $C$ subunits from $R$ subunits and allows PKA to be enzymatically active. We found that three of four FL-HCCs demonstrate a significant increase in cAMP-induced activity compared to the paired nontumor livers (Sample $1-0.79$ vs. $0.44 \mathrm{pmol} / \mathrm{min} / \mathrm{mg}$, sample $2-1.62$ vs. $0.49 \mathrm{pmol} / \mathrm{min} / \mathrm{mg}$, sample $4-1.96$ vs. $1.20 \mathrm{pmol} / \mathrm{min} / \mathrm{mg}$; 

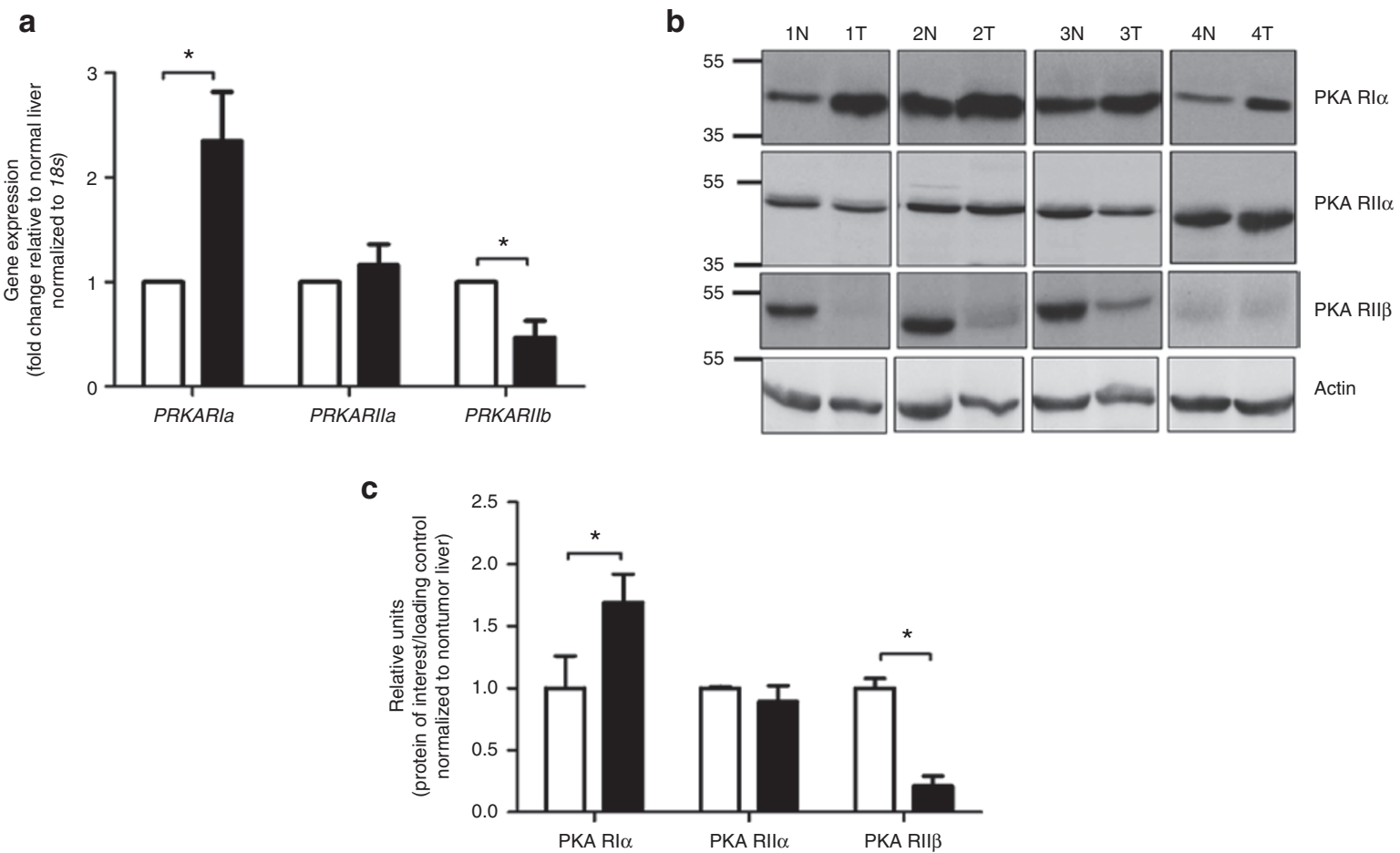

Figure 2. RNA and protein expression of holoenzyme components. (a) Relative expression of PRKARIa, PRKARIla, and PRKARIlb mRNAs in FL-HCC samples $\left(n=4\right.$, black bars) and paired, nontumor liver $\left(n=4\right.$, white bars). ${ }^{P} P<0.05$. (b) Immunoblot analyses for protein kinase $\mathrm{A}(\mathrm{PKA})$ subunit Rl $\alpha$, Rll $\alpha$, and RII $\beta$ proteins in FL-HCC samples $(\mathrm{T})$ and paired, nontumor liver $(\mathrm{N})$. Actin is shown as loading control. (c) Densitometry analysis (ImageJ, NIH) of FL-HCC samples $\left(n=4\right.$, black bars) and paired, nontumor liver $\left(n=4\right.$, white bars) from the immunoblot shown in $\mathbf{b}$. ${ }^{*} P<0.05$. HCC, hepatocellular carcinoma.

a

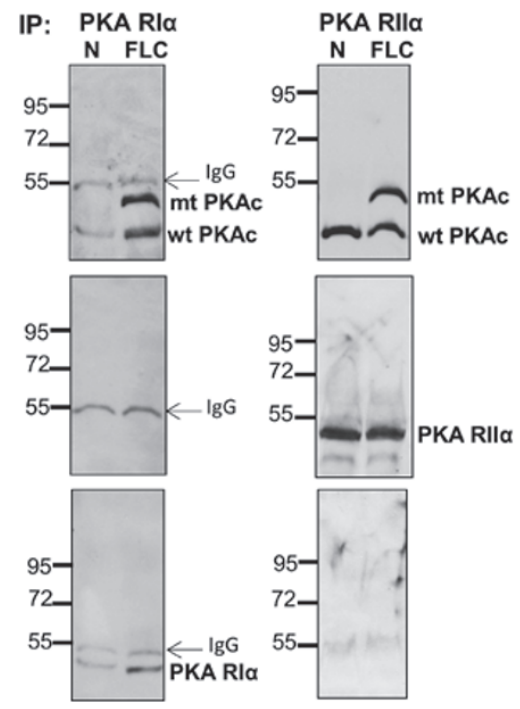

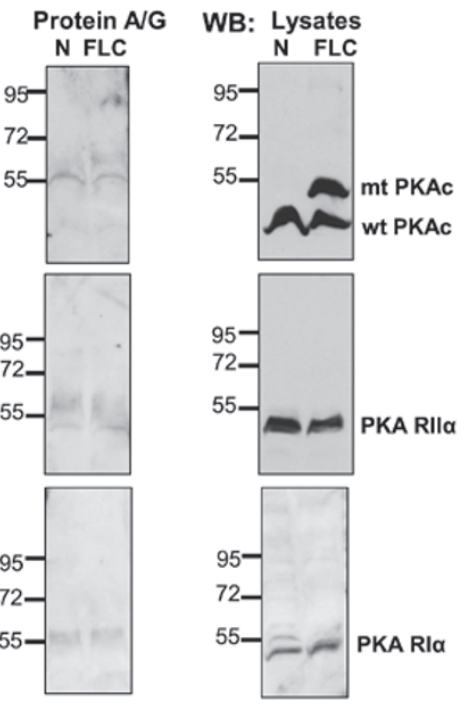

b

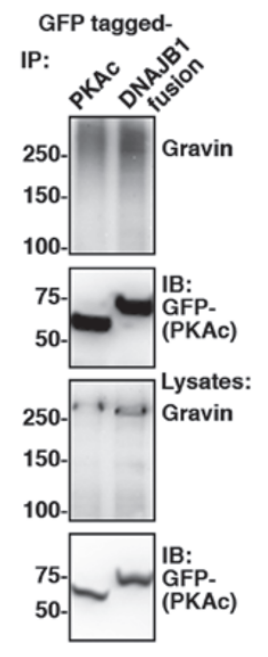

Figure 3. Interaction between PKA R and C subunits. (a) Tumor (FLC) and liver (N) lysates were subjected to immunoprecipitation with antibodies to Rl $\alpha$ and Rll $\alpha$ subunits and immunoblotted for PKA C (top row), Rll $\alpha$ (middle row) and Rl $\alpha$ (bottom row). Bands representing lgG are indicated by arrows. Control IPs without primary antibody are shown in the Protein A/G column, and immunoblots of total cell lysates are represented in the right column. Data are representative of four independent experiments on three different tumors. (b) Protein lysates from HEK 293 cells that were transiently transfected with either YFP-tagged wild-type PKA C or YFP-tagged DNAJB1-PKA C (DNAJB1 fusion) were immunoprecipitated with antibodies for GFP, followed by immunoblotting with antibodies to gravin (top row, left), AKAP 79 (top row, right), and PKA C (second row). Immunoblots performed on nonimmunoprecipitated whole cell lyates are shown as controls (rows 3 and 4). PKA, protein kinase A.

$P$ values all $<0.05)$. Patient number 3 showed less cAMPinduced activity in the FL-HCC compared to its nontumor liver counterpart (0.56 vs. $1.41 \mathrm{pmol} / \mathrm{min} / \mathrm{mg}$; Figure 4, panel a). We then tested the baseline and cAMP-induced PKA activity of three additional FL-HCC samples (two lymph node metastases and one hepatic recurrence) and compared them 


\section{Articles | Riggleet al.}

to the non-tumor liver from a separate patient with FL-HCC. We again found no difference in the baseline PKA activity, but following treatment with cAMP there was an average 3.2-fold increase in PKA activity per microgram of tissue in tumor samples compared to nonpaired liver (Figure 4, panel b). Importantly, addition of the PKI 5-24 peptide, a well characterized and specific inhibitor of PKA (22), completely abolished the detected PKA activity in all samples (Supplementary Figure S1 online).

Next, we performed a dose-response kinase assay using increasing concentrations of cAMP, and confirmed increased cAMP-induced activity in one FL-HCC compared to its paired nontumor liver (Figure 5, panel a). While the total enzymatic activity in this FL-HCC was twofold greater than its paired normal liver, the calculated $\mathrm{K}_{\mathrm{m}}$ for PKA activation are $68.8 \pm 22.3$ (95\% CI: $6.0-130.7)$ and $36.2 \pm 22.04$ (95\% CI: $0.0-97.4)$, respectively $(P>0.05)$ (Figure 5, panel b). These data suggest that the intrinsic kinase activities are similar in FL-HCC and nontumor liver, and that the increased absolute PKA activities in tumors may thus be attributed to greater enzyme abundance.

\section{Localization of Regulatory and Catalytic Subunits}

Upon binding of cAMP to the R subunits, the PKA holoenzyme dissociates in order to permit active $\mathrm{C}$ subunit to phosphorylate effector proteins. Moreover, the subcellular location of the PKA holoenzyme is constrained by a family of AKAPs that tether the enzyme in proximity to selected substrates (23). To determine the subcellular distribution of PKA RII $\alpha$ and PKA C subunits in FL-HCC, we performed triple immunofluorescent (IF) labeling on formalin-fixed tissues using antibodies to PKA RIIo, PKA C, and 4',6-diamidino-2-phenylindole (DAPI) (nuclear stain). We found that in both FL-HCCs and normal liver, PKA C and PKA RII $\alpha$ primarily co-localize in the cytoplasm (Figure 6, panel a). When we quantified the number of hepatocytes in which there was overlapping PKA C and DAPI signals, suggesting nuclear localization of PKA C, we found that there were "positive" nuclear signals in $3.1 \pm 0.7 \%$ of tumor cells in FL-HCCs and $3.7 \pm 0.4 \%$ of hepatocytes in normal livers (Figure 6, panel b). These data suggest that the mutant PKA C in FL-HCC is predominately localized to the cytoplasm, similar to that of the WT protein.

\section{DISCUSSION}

Our findings support the notion that aberrant PKA activity plays a causative role in FL-HCC, and represent the first study to demonstrate enhanced cAMP stimulated PKA activity in human FL-HCC tissues. The oncogenic activity of PKA signaling has been described in human endocrine tumors, including cortisol producing adrenal adenomas and those associated with Carney's syndrome (growth hormone-secreting pituitary adenomas, thyroid, and testicular tumors) $(24,25)$. The effects of PKA signaling on cell biology are highly pleiotropic and dependent on cell context and subcellular location $(18,26)$. For the most part, PKA inhibits proliferation and promotes differentiated cellular functions, but in certain cells like
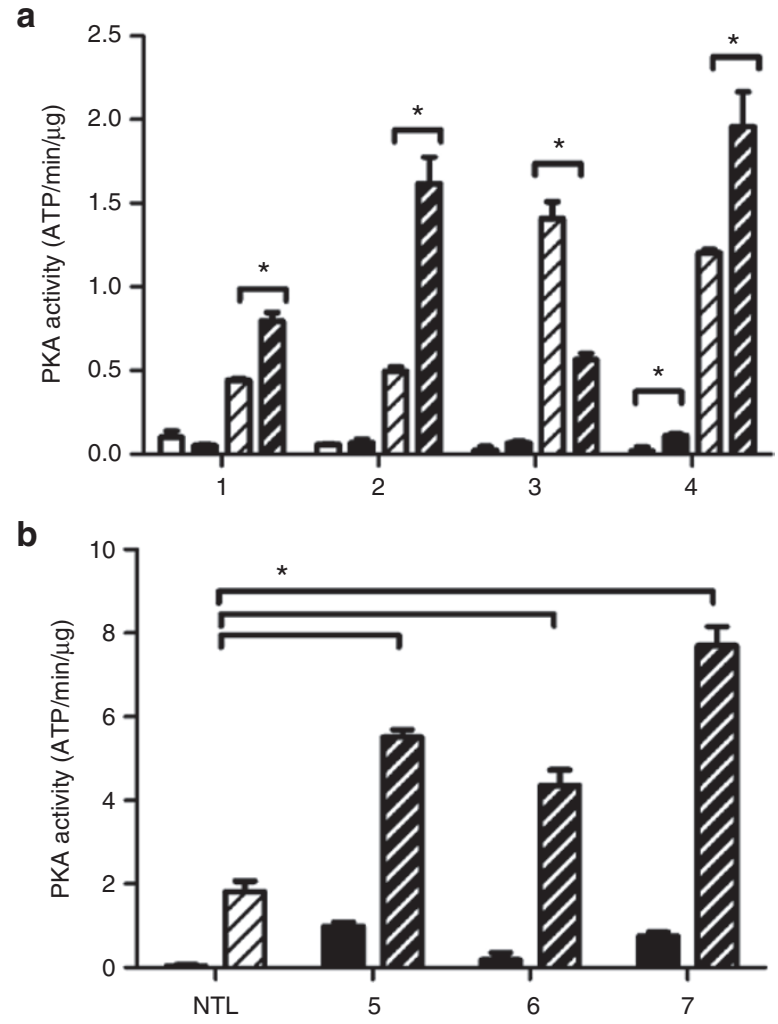

Figure 4. Enhanced CAMP-induced kinase activity in FL-HCC. (a) Protein kinase A (PKA) kinase activities of four separate FL-HCCs (black bars) compared to paired, normal livers (white bars) with (striped bars) or without (solid bars) CAMP stimulation, ${ }^{*} P<0.05$. (b) PKA kinase activities in the presence (solid bars) or absence (striped bars) of CAMP of three additional FL-HCC's (black bars) from two lymph node metastases (samples 5 and 6) and one local tumor recurrence (sample 7) compared with an unrelated nontumor liver (white bars) of a FL-HCC patient. ${ }^{*} P<0.05$. Data are representative of three independent experiments performed with each tissue lysate. HCC, hepatocellular carcinoma.

somatotrophs, PKA activity induces cell division in response to growth hormone. Similarly, inactivation of PRKARIA in GH3 cells, which would enhance PKA activity, stimulates cell proliferation and increases cyclin D1 and ERK activity $(27,28)$. We failed to find evidence of elevated ERK phosphorylation in FL-HCCs (Supplementary Figure S2 online), but instead uncovered consistent mTORC1 activity in these tumors (29). A relationship between cAMP/PKA signaling and mTORC1 activity has been reported, but these findings have not been consistent and vary with differences in cell lineage (30). We await the final analyses of a clinical trial evaluating the efficacy of an mTORC1 inhibitor in FL-HCC patients (NCT01642186).

Using a panel of human primary liver tumors, we demonstrate that the DNAJB1-PRKACA chimeric transcript and resultant fusion protein are uniquely and uniformly found in FL-HCCs. How this genomic rearrangement causes hepatocyte transformation in an otherwise normal, noncirrhotic liver remains to be elucidated. Two obvious possibilities include dysregulation of PKA signaling and loss of a DNAJB1 associated function. To begin to address the former hypothesis, previous in vitro work demonstrated that the DNAJB1-PRKACA protein product 

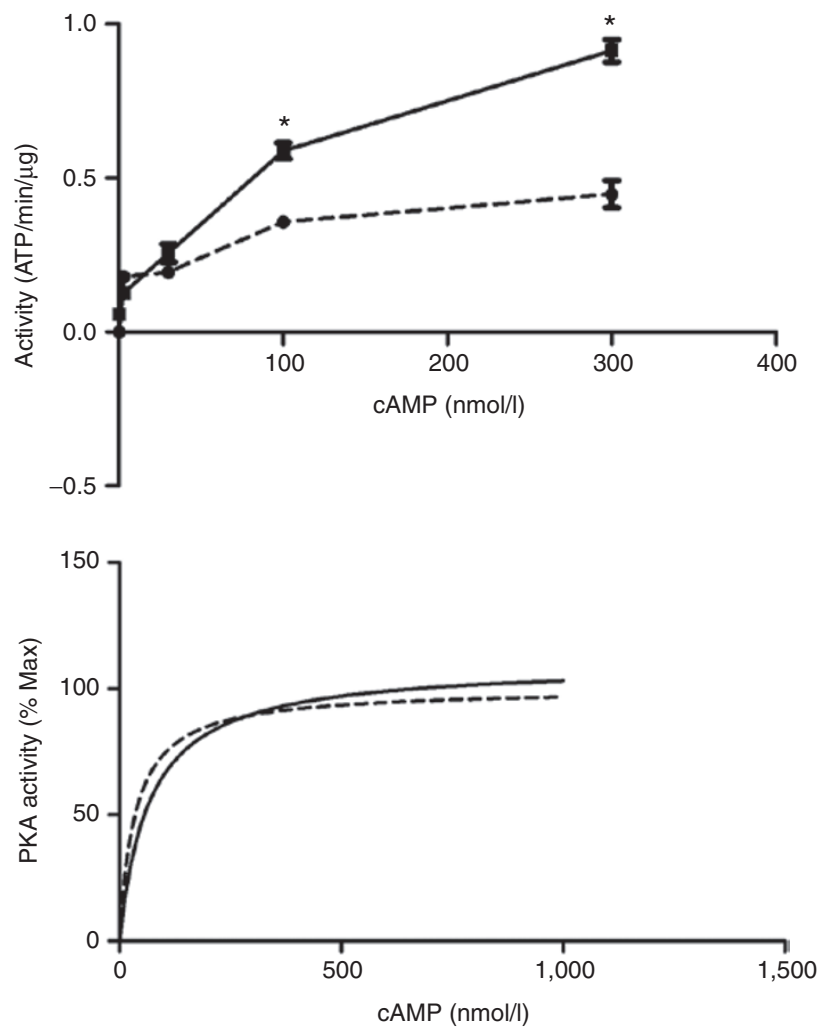

Figure 5. Dose response of CAMP-dependent protein kinase A (PKA) activity. (a) CAMP-dependent dose response in PKA activity in an FL-HCC (solid line) compared with its paired nontumor liver (dashed line). PKA activities in FL-HCC compared to nontumor liver at CAMP concentrations of $0.3 \mathrm{nmol} / \mathrm{l}$ ( $0.13 \mathrm{vs} .0 .18 \mathrm{ATP} / \mathrm{min} / \mathrm{ug}), 30 \mathrm{nmol} / \mathrm{l}$ ( $0.25 \mathrm{vs} .0 .19$ ATP/ $\mathrm{min} / \mu \mathrm{g}), 100 \mathrm{nmol} / \mathrm{l}(0.59 \mathrm{vs} .0 .36 \mathrm{ATP} / \mathrm{min} / \mu \mathrm{g})$, and $300 \mathrm{nmol} / \mathrm{l}(0.91 \mathrm{vs}$. $0.45 \mathrm{ATP} / \mathrm{min} / \mu \mathrm{g}$ ), ${ }^{*} P<0.05$. (b) Nonlinear regression curve based on the Michaelis-Menten equation for the calculation of $\mathrm{K}_{m} . \mathrm{K}_{\mathrm{m}}$ was calculated as $68.8 \pm 22.3$ (95\% Cl: 6.0-130.7) and 36.2 \pm 22.04 (95\% Cl: $0.0-97.4)$ in the FL-HCC (solid line) and paired nontumor liver (dashed line), respectively $(P>0.05)$. HCC, hepatocellular carcinoma.

retains kinase activity, but its activity in human FL-HCCs had not been examined. In this study, we examined a series of FL-HCCs and detected elevated kinase activity in most tumors compared to paired nontumor livers, implying an oncogenic function of the mutant PKA fusion enzyme. Importantly, PKA activity in human FL-HCC is cAMP-dependent, and while the basal activity in tumors is unchanged from that of nontumor liver, FL-HCCs have significantly greater capacity for PKA activation in the presence of cAMP. This finding is consistent with a model in which the mutant PKA C interacts with the $\mathrm{R}$ subunits to create a functional holoenzyme that responds to second messengers, but may have different avidity for specific $\mathrm{R}$ isoforms.

Our in vivo data from patient samples are supported by elegant in vitro studies by Cheung et al. (9), which were published during the preparation of this manuscript. Their group determined the crystal structure of the DNAJB1-PRKACA protein product, confirmed that it could bind $\mathrm{R}$ subunits, and posited that increased PKA activity in FL-HCCs is due to increased expression of $\mathrm{C}$ subunit off the DNAJB1 promoter. In keeping with Cheung et al.'s in vitro work, we found elevated PKA activity in FL-HCCs, which could result from a number of causes. Possible explanations include an increase in expression of the fusion transcript beyond that of either WT PRKACA or DNAJB1. Although the 5' promoter sequence of the chimeric gene is in common with WT DNAJB1, the mutant transcript is 3.8 -fold more abundant than that of the WT allele. We speculate that loss of the 3' UTR of DNAJB1 in the mutant allele could result in increased mRNA stability. Alternatively, elements within the $\sim 400 \mathrm{~kb}$ deletion may influence the promoter activity and transcriptional regulation of DNAJB1-PRKACA. Another possible explanation for the enhanced sensitivity to cAMP in FL-HCCs could be allosteric hindrance by the larger fusion protein, resulting in reduced stability of the heterotetramer in the presence of cAMP. We found that the $\mathrm{K}_{\mathrm{m}} \mathrm{s}$ for activation of PKA in FL-HCC and paired normal liver samples are similar, however, which is supported by in vitro studies showing that intrinsic PKA kinase activity is not altered by the mutation (9). Honeyman et al. similarly reported no difference in the activities of the mutant and WT PKA C when overexpressed in 293 cells (7). In addition, our data presented in Figure 3b argue that at least in the context of the PKA holoenzymes, the mutant DNAJ-PKA C fusion does not interfere with association with the anchoring proteins Gravin or AKAP79. A logical extension of this finding would be to propose that DNAJ-PKA C fusion is compartmentalized to the membranes and cortical cytoskeleton in FL-HCC tumor cells by virtue of its ability to associate with these AKAPs (31-34). Our notion of normal subcellular distribution of DNAJ-PKA C fusion in FL-HCCs is also consistent with the IF data presented in Figure 6, which demonstrate that the mutant kinase does not accumulate in the nucleus of tumor cells.

Importantly, we found that increased PKA activity in FL-HCCs is dependent on elevation of intracellular cAMP. This finding suggests that aberrant PKA signaling in FL-HCC would be dependent upon a renewable supply of second messengers. One potential source of increased cAMP is aberrant activation of upstream G-protein coupled receptors (GPCRs) and $\mathrm{G}$ proteins such as $\mathrm{G}_{\alpha \mathrm{s}}$, which are known to stimulate adenylyl cyclases to increase cAMP synthesis (35). The neurotensin family of GPCRs is one possible candidate driver; the association between neurotensin and FL-HCC was first demonstrated in 1984, but its molecular significance has not yet been established (36). Collectively, our results when considered with recent finding from other laboratories extend the argument that the DNAJ-PKA C fusion drives the development of FL-HCC through unknown phosphorylation events in the cytoplasm.

Future investigations of the function of DNAJB1-PRKACA in nontransformed hepatocytes will be critical for enhancing our understanding of the pathogenesis of FL-HCC. The roles of the first exon of DNAJB1 in the context of the fusion transcript and other potential secondary drivers of FL-HCC, such as the CLPTM1L-GLIS3 mutation reported by $\mathrm{Xu}$ et al. (8), also remain to be defined. We anticipate that mapping out the effector pathways downstream of amplified PKA signaling in FL-HCC will lead to the development of targeted therapies for patients with this disease. 


\section{Articles $\mid$ Riggleetal.}
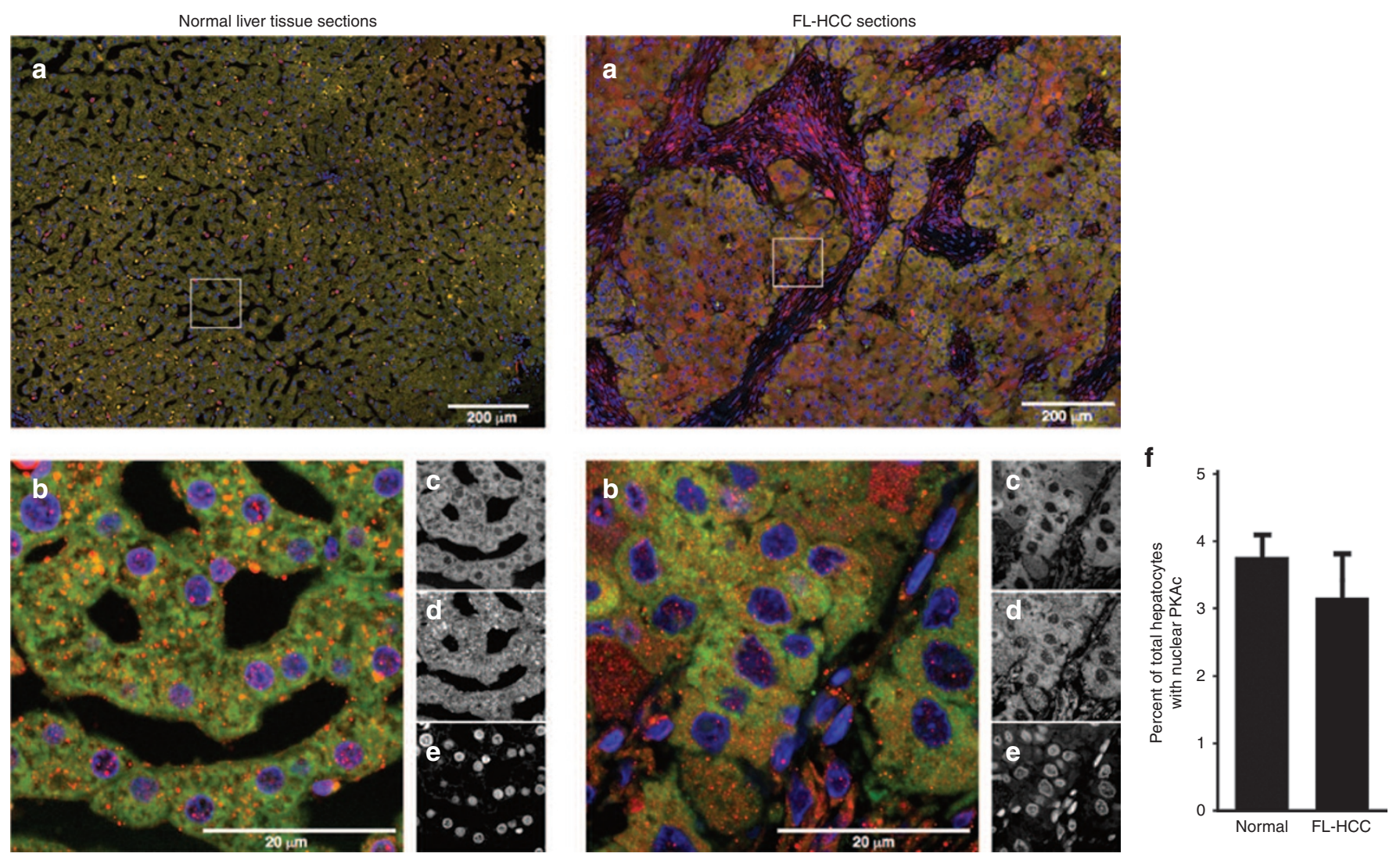

Figure 6. Localization of protein kinase A (PKA) subunits. Triple immunofluorescence photomicrographs of an FL-HCC (left) vs. normal liver (right). Images stained with antibodies against PKA C (c) PKA Rlla (d), and DAPI (e) are shown in grayscale, along with merged images with PKA C shown in green, PKA Rlla in red, and DAPI in blue (b). All images were obtained at 63× magnification; scale bars represent 200 microns. Composites of high-resolution sets of images representing adjacent fields are shown as wide-field microscopy (a). (f) Four separate FL-HCCs and four nontumor livers were analyzed for overlapping DAPI and PKA C signal and the percentage of cells with "nuclear" PKA C signals was found to be $3.1 \pm 0.7 \%$ of tumor cells in FL-HCCs and $3.7 \pm 0.4 \%$ of hepatocytes in normal livers $(P>0.05)$. HCC, hepatocellular carcinoma.

\section{METHODS}

\section{Human Liver Samples}

Human FL-HCCs with paired normal liver, two lymph node metastases, and a liver recurrence were obtained from the University of Washington Medical Center and Seattle Children's Hospital, after obtaining Institutional Review Board approval (SCH IRB \#14393). Other types of liver tumors ("classic" HCCs (four HCC secondary to HCV cirrhosis, one secondary to HBV cirrhosis, and five secondary to cirrhosis of unknown origin] and cholangiocarcinomas) were obtained from the University of Washington Medical Center (UW IRB \#31281). Informed consent was obtained from the subject and/or parent prior to tumor resection.

\section{RNA Extraction and RT-qPCR}

RNA was extracted from tumors and surrounding liver tissue using TRIzol (Life Technologies, Grand Island, NY) as described by the manufacturer. qRT-PCR reactions contained cDNA synthesized from $1.0 \mu \mathrm{g}$ RNA using MMLV reverse transcriptase (Life Technologies), and either SYBR green (Agilent Technologies, Santa Clara, CA) or TaqMan (Life Technologies) master mix. Primers and their sequences are listed in the supplementary data table (Supplementary Table S1 online). Cycling conditions were $95{ }^{\circ} \mathrm{C}$ for $10 \mathrm{~min}$, and 39 cycles of $95^{\circ} \mathrm{C}$ for $15 \mathrm{~s}$, and $60{ }^{\circ} \mathrm{C}$ for $60 \mathrm{~s}$, with a final extension at $72^{\circ} \mathrm{C}$ for 1 min using a RotorGene 3000 thermocycler (Corbett Research, San Francisco, CA). Data are represented as delta delta $C_{t}$ values after normalization to GAPDH (SYBR green) or $18 S$ (TaqMan) mRNA levels.

\section{Immunoblotting}

Human FL-HCCs and normal liver samples were homogenized in icecold radioimmunoprecipitation buffer containing protease inhibitors, and protein concentrations were measured using the BCA Protein Assay (Pierce, Rockford, IL). Equal amounts of protein were separated by sodium dodecyl sulfate - poly-acrylamide gel electrophoresis (SDS-PAGE), transferred to Immobilin-P membranes (Millipore, Bedford, MA) and incubated at $4{ }^{\circ} \mathrm{C}$ overnight with the following primary antibodies: PKA C (SC-903, Santa Cruz Biotechnologies, Santa Cruz, CA), PKA RII $\alpha$ (SC-909, Santa Cruz Biotechnologies), PKA RII $\beta$ (610626, BD Biosciences, San Jose, CA), p-p44/42 MAPK (Erk1/2) (Thr202/Tyr204), total p44/42 MAPK (Erk1/2), and PKA RI $\alpha$ (9101, 9102, 5675, Cell Signaling, Danvers, MA), and $\beta$-Actin (A5441, Sigma, St. Louis, MO). Densitometry analysis was performed using NIH ImageJ software (National Institutes of Health, Bethesda, $\mathrm{MD}$ ) by measuring the integrated density around each band and normalizing to the integrated density of the actin band for each sample.

\section{Immunoprecipitation}

A human FL-HCC and paired non-tumor liver were homogenized in $1 \%$ Triton-X buffer (20 mmol/l 4-(2-hydroxyethyl)-1-piperazineethanesulfonic acid ( $\mathrm{pH} 7.4), 150 \mathrm{mmol} / \mathrm{l} \mathrm{NaCl}, 5 \mathrm{mmol} / \mathrm{l}$ Ethylenediaminetetraacetic acid (EDTA), and 1\% Triton with protease inhibitors). $600 \mu \mathrm{g}$ of protein $(1 \mu \mathrm{g} / \mu \mathrm{l})$ per sample was precleared with Pansorbin (Millipore) for $1 \mathrm{~h}$ and incubated overnight with PKA RI $\alpha$ (610610, BD Biosciences) or PKA RII $\alpha$ antibody (as described above). Lysates were then incubated with either Protein A or Protein G Sepharose beads (Sigma Aldrich) for $1 \mathrm{~h}$. Beads were then washed with $1 \%$ Triton-X buffer. After heating in sample buffer each sample (normal and tumor) was divided equally and loaded identically on three SDS-PAGE gels (8 or 10\%). Immunoprecipitated samples were immunoblotted with antibodies against PKARI $\alpha$ (5675, Cell Signaling), PKA C (SC-903), and PKA RII $\alpha$ (SC-909) (Santa 
Cruz Biotechnologies). Primary antibodies were detected with AntiRabbit IgG-HRP (GE Healthcare, UK) or Protein-A HRP (Pierce Biotechnology).

\section{AKAP Binding}

YFP-tagged WT PKA C and YFP tagged DNAJ-PKA C were constructed using Vivid Colors pcDNA6.2/C-EmYFP-DEST Vector (v357-20, Thermo Scientific, Waltham, MA) according to the manufacturer's protocol. Transient transfections were performed in HEK293 cells with $8 \mathrm{ng}$ YFP-WT PKAC or $5 \mathrm{ng}$ YFP-DNAJ-PKAC and Lipofectamine 2000 reagent (11668027, Thermo Scientific). After $48 \mathrm{~h}$ of expression, cells were lysed using $20 \mathrm{mmol} / \mathrm{l}$ Tris- $\mathrm{HCl} \mathrm{pH8}$; $30 \mathrm{mmol} / \mathrm{l} \mathrm{NaCl} ; 0.02 \%$ Triton-X-100; 0.02\% Na-DOC; $0.01 \%$ SDS; $1 \mathrm{mmol} / \mathrm{l} \mathrm{EDTA} ; 1 \mathrm{mmol} / \mathrm{l} \mathrm{MgCl}_{2}$. Cell lysates were cleared and then incubated with antibodies against Green fluorescent protein (GFP) (A-11122; Life Technologies) overnight. Lysates were incubated with $20 \mu$ Protein G Sepharose beads (Sigma Aldrich) for $1 \mathrm{~h}$, and beads were washed with $1 \%$ Triton-X buffer five times. After heating in sample buffer each sample was divided equally and loaded identically on SDS-PAGE gels. Immunoprecipitated samples were immunoblotted with antibodies against AKAP 79 (ABS102, Millipore), Gravin (g3795, Sigma Aldrich), or with the GFP antibody listed above as a control. Primary antibodies were then detected as described above.

\section{Immunofluorescence}

Triple-immunofluorescent labeling was performed on formalinfixed normal liver sections using conjugated antibodies to the PKA C subunit (610981, BD Biosciences), PKA RII $\alpha$ subunit (SC-909, Santa Cruz Biotechnologies), and DAPI (62248, Thermo Scientific), as described (37). Images were collected on a Zeiss LSM 510 confocal microscope (Carl Zeiss, Oberkochen, Germany). Quantification of PKA C signal in the nucleus was performed using merged images and counting the number of hepatocytes with overlapping green (PKA C) and blue (DAPI) signals. Five hundred total hepatocytes were counted for each sample.

\section{PKA Activity Assay}

Tumor and liver lysates were subjected to radioactive kinase activity assays, which were performed in triplicate using Kemptide as a substrate and ${ }^{32} \mathrm{P} \gamma$-ATP, either with or without incubation with cAMP ( $5 \mu \mathrm{mol} / 1$, to maximally stimulate PKA) and/or PKI, a specific inhibitor of PKA as described (21). Dose-response experiments were performed in triplicate using cAMP concentrations of 30,100,300, and $1,000 \mathrm{nmol} / \mathrm{l}$. $\mathrm{K}_{\mathrm{m}}$ calculations were performed using GraphPad Prism (GraphPad Software, San Diego, CA) and the Michaelis-Menten formula (38).

\section{Statistical Analyses}

Statistical analysis was performed using GraphPad Prism (GraphPad Software) on all data, using the paired Student $t$-test and analysis of variance with post hoc test where appropriate, with a $P$ value $\leq 0.05$ considered to be significant.

\section{SUPPLEMENTARY MATERIAL}

Supplementary material is linked to the online version of the paper at http:// www.nature.com/pr

\section{ACKNOWLEDGMENTS}

The authors would like to thank Lorene Langeberg for her work helping to construct the figures for the manuscript.

\section{STATEMENT OF FINANCIAL SUPPORT}

American Surgical Association Foundation, Beverly, MA (K.J.R.), the University of Washington Department of Surgery Research Reinvestment Fund, Seattle, WA (K.J.R. and R.S.Y.), the Herbert S. Coe Foundation, Seattle, WA (K.J.R.), the Howard Hughes Medical Institute, Chevy Chase, MD (J.D.S., R.E.T., M.W., and B.S.), and National Institutes of Health grants DK54441 and DK105542 (J.D.S., B.S., and M.W.). R.E.T. is supported by Interdisciplinary Training Grant in Cancer, T32 CA080416.

Disclosure: The authors have no financial conflicts of interest to disclose.

\section{REFERENCES}

1. Nordenstedt H, White DL, El-Serag HB. The changing pattern of epidemiology in hepatocellular carcinoma. Dig Liver Dis 2010;42 Suppl 3:S206-14.

2. Hoshida Y. Molecular signatures and prognosis of hepatocellular carcinoma. Minerva Gastroenterol Dietol 2011;57:311-22.

3. Kan Z, Zheng H, Liu X, et al. Whole-genome sequencing identifies recurrent mutations in hepatocellular carcinoma. Genome Res 2013;23:142233.

4. Qi LN, Li LQ, Chen YY, et al. Genome-wide and differential proteomic analysis of hepatitis B virus and aflatoxin B1 related hepatocellular carcinoma in Guangxi, China. PLoS One 2013;8:e83465.

5. Mayo SC, Mavros MN, Nathan H, et al. Treatment and prognosis of patients with fibrolamellar hepatocellular carcinoma: a national perspective. J Am Coll Surg 2014;218:196-205.

6. Torbenson M. Fibrolamellar carcinoma: 2012 update. Scientifica (Cairo) 2012;2012:743790.

7. Honeyman JN, Simon EP, Robine N, et al. Detection of a recurrent DNAJB1-PRKACA chimeric transcript in fibrolamellar hepatocellular carcinoma. Science 2014;343:1010-4.

8. Xu L, Hazard FK, Zmoos AF, et al. Genomic analysis of fibrolamellar hepatocellular carcinoma. Hum Mol Genet 2015;24:50-63.

9. Cheung J, Ginter C, Cassidy M, et al. Structural insights into mis-regulation of protein kinase A in human tumors. Proc Natl Acad Sci USA 2015;112:1374-9.

10. Amieux PS, McKnight GS. The essential role of RI alpha in the maintenance of regulated PKA activity. Ann N Y Acad Sci 2002;968:75-95.

11. Uhler MD, McKnight GS. Expression of cDNAs for two isoforms of the catalytic subunit of cAMP-dependent protein kinase. J Biol Chem 1987;262:15202-7.

12. Dell'Acqua ML, Scott JD. Protein kinase A anchoring. J Biol Chem 1997;272:12881-4

13. Scott JD, Pawson T. Cell signaling in space and time: where proteins come together and when they're apart. Science 2009;326:1220-4.

14. Pritchard CC, Salipante SJ, Koehler K, et al. Validation and implementation of targeted capture and sequencing for the detection of actionable mutation, copy number variation, and gene rearrangement in clinical cancer specimens. J Mol Diagn 2014;16:56-67.

15. Newlon MG, Roy M, Hausken ZE, Scott JD, Jennings PA. The A-kinase anchoring domain of type IIalpha cAMP-dependent protein kinase is highly helical. J Biol Chem 1997;272:23637-44.

16. Wu J, Brown SH, von Daake S, Taylor SS. PKA type IIalpha holoenzyme reveals a combinatorial strategy for isoform diversity. Science 2007;318:274-9.

17. Zick SK, Taylor SS. Interchain disulfide bonding in the regulatory subunit of cAMP-dependent protein kinase I. J Biol Chem 1982;257:2287-93.

18. Scott JD, Stofko RE, McDonald JR, Comer JD, Vitalis EA, Mangili JA. Type II regulatory subunit dimerization determines the subcellular localization of the cAMP-dependent protein kinase. J Biol Chem 1990;265:21561-6.

19. Carnegie GK, Means CK, Scott JD. A-kinase anchoring proteins: from protein complexes to physiology and disease. IUBMB Life 2009;61:394-406.

20. Smith FD, Reichow SL, Esseltine JL, et al. Intrinsic disorder within an AKAP-protein kinase A complex guides local substrate phosphorylation. Elife 2013;2:e01319.

21. Scott JD, Fischer EH, Demaille JG, Krebs EG. Identification of an inhibitory region of the heat-stable protein inhibitor of the cAMP-dependent protein kinase. Proc Natl Acad Sci USA 1985;82:4379-83.

22. Scott JD, Glaccum MB, Fischer EH, Krebs EG. Primary-structure requirements for inhibition by the heat-stable inhibitor of the cAMP-dependent protein kinase. Proc Natl Acad Sci USA 1986;83:1613-6.

23. Welch EJ, Jones BW, Scott JD. Networking with AKAPs: context-dependent regulation of anchored enzymes. Mol Interv 2010;10:86-97.

24. Beuschlein F, Fassnacht M, Assié G, et al. Constitutive activation of PKA catalytic subunit in adrenal Cushing's syndrome. N Engl J Med 2014;370:1019-28

25. Kirschner LS, Carney JA, Pack SD, et al. Mutations of the gene encoding the protein kinase A type I-alpha regulatory subunit in patients with the Carney complex. Nat Genet 2000;26:89-92. 


\section{Articles | Riggle et al.}

26. Carnegie GK, Scott JD. A-kinase anchoring proteins and neuronal signaling mechanisms. Genes Dev 2003;17:1557-68.

27. Lania AG, Mantovani G, Ferrero S, et al. Proliferation of transformed somatotroph cells related to low or absent expression of protein kinase a regulatory subunit 1A protein. Cancer Res 2004;64:9193-8.

28. Mantovani G, Bondioni S, Ferrero S, et al. Effect of cyclic adenosine 3,'5'-monophosphate/protein kinase a pathway on markers of cell proliferation in nonfunctioning pituitary adenomas. J Clin Endocrinol Metab 2005;90: $6721-4$.

29. Riehle KJ, Yeh MM, Yu JJ, et al. mTORC1 and FGFR1 signaling in fibrolamellar hepatocellular carcinoma. Mod Pathol 2015;28:103-10.

30. Pringle DR, Vasko VV, Yu L, et al. Follicular thyroid cancers demonstrate dual activation of PKA and mTOR as modeled by thyroid-specific deletion of Prkarla and Pten in mice. J Clin Endocrinol Metab 2014;99: E804-12.

31. Canton DA, Keene CD, Swinney K, et al. Gravin is a transitory effector of polo-like kinase 1 during cell division. Mol Cell 2012;48:547-59.
32. Carnegie GK, Soughayer J, Smith FD, et al. AKAP-Lbc mobilizes a cardiac hypertrophy signaling pathway. Mol Cell 2008;32:169-79.

33. Langeberg LK, Scott JD. Signalling scaffolds and local organization of cellular behaviour. Nat Rev Mol Cell Biol 2015;16:232-44.

34. Smith FD, Langeberg LK, Cellurale C, et al. AKAP-Lbc enhances cyclic AMP control of the ERK1/2 cascade. Nat Cell Biol 2010;12: $1242-9$.

35. Sunahara RK, Dessauer CW, Whisnant RE, Kleuss C, Gilman AG. Interaction of Gsalpha with the cytosolic domains of mammalian adenylyl cyclase. J Biol Chem 1997;272:22265-71.

36. Collier NA, Weinbren K, Bloom SR, Lee YC, Hodgson HJ, Blumgart LH. Neurotensin secretion by fibrolamellar carcinoma of the liver. Lancet 1984;1:538-40.

37. Dan YY, Riehle KJ, Lazaro C, et al. Isolation of multipotent progenitor cells from human fetal liver capable of differentiating into liver and mesenchymal lineages. Proc Natl Acad Sci USA 2006;103:9912-7.

38. Voet D, Voet JG. Biochemistry. 3rd edn. New York, NY: Wiley, 2004:480. 